\title{
Measuring the Mean Inner Potential Of Bernal Graphite Using Off-axis Electron Holography
}

Avi Auslender ${ }^{1}$, George Levi ${ }^{2}$, Vladimir Ezersky ${ }^{3}$, Semën Gorfman $^{4}$, Oswaldo Diéguez ${ }^{4}$ and Amit Kohn ${ }^{4}$

${ }^{1}$ Department of Materials Science and Engineering, The Iby and Aladar Fleischman Faculty of Engineering, Tel Aviv University, Tel Aviv 6997801, Israel, Tel Aviv, Tel Aviv, Israel, ${ }^{2}$ Department of Materials Science and Engineering, The Iby and Aladar Fleischman Faculty of Engineering, Tel Aviv University, Tel Aviv 6997801, Israel, Tel Aviv, Israel, ${ }^{3}$ Department of Materials Engineering, Ben-Gurion University of the Negev, Beer-Sheva 84105, Israel, Beer-Sheva, HaDarom, Israel, ${ }^{4}$ Department of Materials Science and Engineering, The Iby and Aladar Fleischman Faculty of Engineering, Tel Aviv University, Tel Aviv 6997801, Israel, United States

The mean-inner-potential (MIP) of a crystal is the average electrostatic Coulomb potential within a crystal with respect to vacuum. The MIP is a fundamental material property which reflects chemical-bonding and crystallographic surfaces [1].

We conducted off-axis electron holography experiments on highly-oriented-pyrolytic-graphite (HOPG) in a transmission-electron-microscope to measure the MIP from nanometer-scale volumes of Bernal graphite oriented with respect to the electron beam, along the principal axis or directions in the basal plane. These MIP were related to mean orbital electron radii and diamagnetic susceptibilities in perpendicular planes. Such intrinsic property measurements are challenging because of defect.induced interfaces at basal planes $[2,3]$. Indeed, our structural examination of HOPG show stacking faults and planar rotations around the principal-axis, such that measuring intrinsic properties requires probing a volume of 102102102nm3.

Experiments on individual Bernal graphite crystals with (0001) basal, or (1-100), (2-1-10) prismatic planes, resulted in MIP of $10.16 \pm 0.40 \mathrm{~V}, 11.37 \pm 0.35 \mathrm{~V}, 12.66 \pm 0.41 \mathrm{~V}$, respectively [4]. First-principles calculations from crystalline slabs [4] confirm these anisotropic measurements with $11.72 \mathrm{~V}, 13.65 \mathrm{~V}$, $14.56 \mathrm{~V}$, respectively. Additionally, these experiments enabled to measure the mean free path for inelastic scattering in graphite of $197 \mathrm{keV}$ electrons at a collection angle of $18 \mathrm{mrad}$ resulting in $150.6 \pm 2.0 \mathrm{~nm}$.

These measured MIP enable to determine projected mean radii of electron orbitals and volume susceptibilities (SI), assuming spherically symmetric charge distribution, at $0.704 \pm 0.015 \AA$, ($1.99 \pm 0.08) 10-5 ; 0.744 \pm 0.015 \AA,(-2.23 \pm 0.07) 10-5 ; 0.785 \pm 0.015 \AA,(-2.48 \pm 0.08) 10-5$.

The measured orbital radii and diamagnetism in the basal plane are comparable to expected values for carbon $\sigma$-bond hybridization [5-7]. Increased MIP on prismatic planes is related to s-orbital components, which decrease due to delocalized electrons between basal planes. 

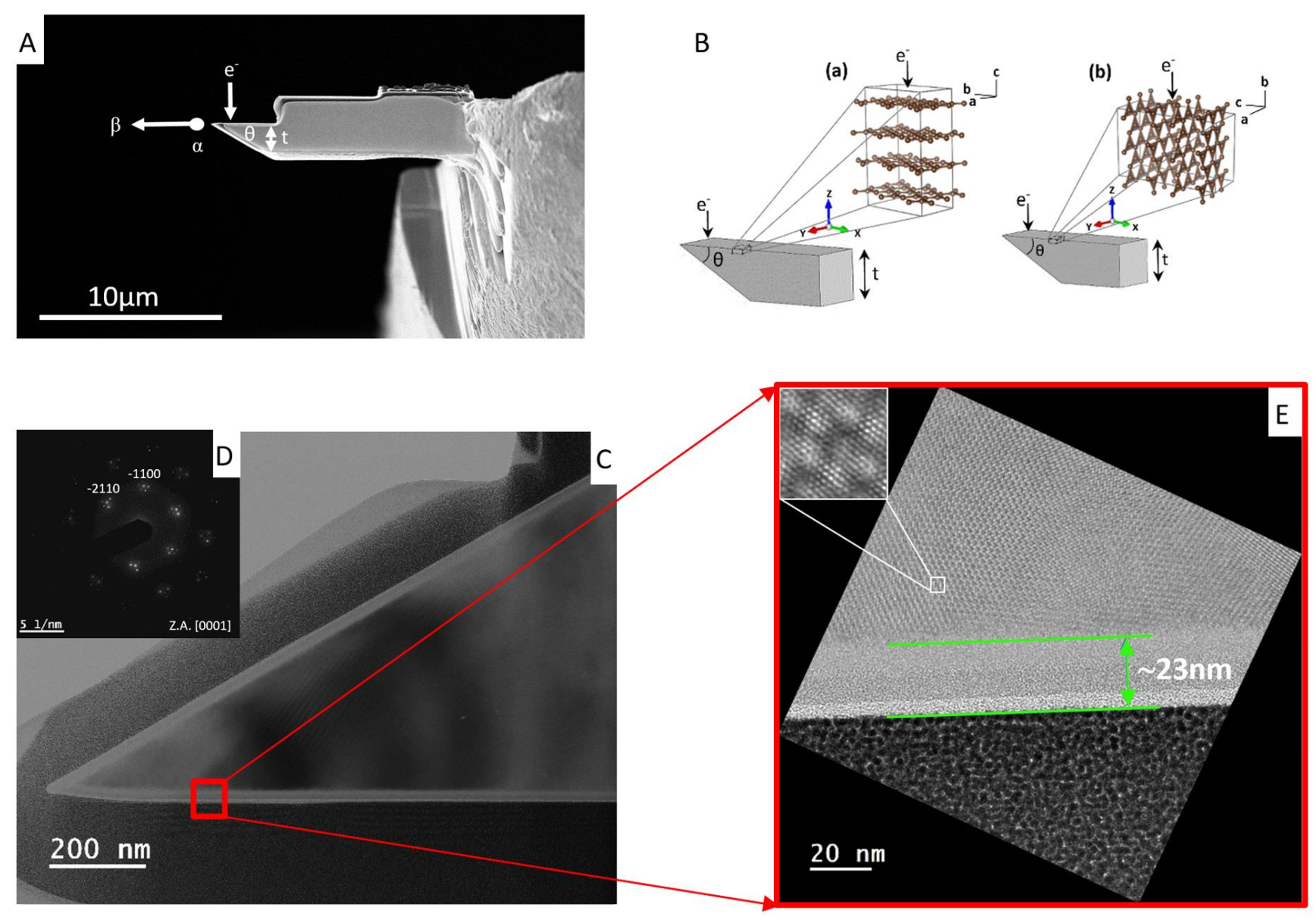

Figure 1. Fig.1 (A) Secondary electron SEM micrograph of a (0001) $30^{\circ}$ wedge sample connected to a $\mathrm{Cu}$ grid and after ion milling illustrating tilt axes, $\alpha, \beta$. t is the sample thickness, $\theta$ is the wedge angle (in this report: $\sim 30^{\circ}$ or $\sim 45^{\circ}$ ). The direction of the electron beam is denoted by the arrow. (B) Schematic illustration of HOPG TEM wedge samples in two crystallographic plane orientations at the surface, where the electron beam direction is denoted by an arrow (z-direction); Electron beam perpendicular B(a) (x-y plane), and parallel B(b) (y-z plane, in the [2-1-10] direction) to (0001) basal plane. (C) Bright field crosssectional TEM micrograph of the wedge sample, from which an angle of $30.5^{\circ}$ is measured, (D) SAED pattern recorded from this sample, edge-on view with respect to the basal plane (E) High-resolution (phase contrast) TEM micrograph from the region denoted schematically by the blue square. The inset shows a magnification of the marked area. 

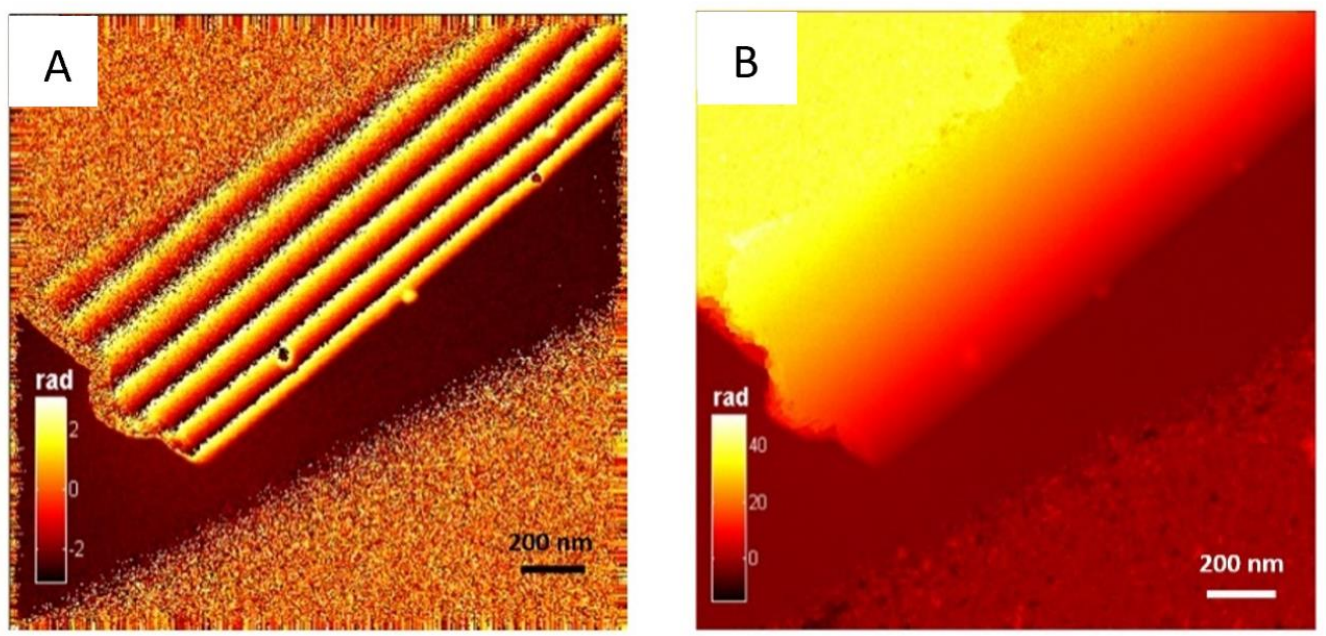

C

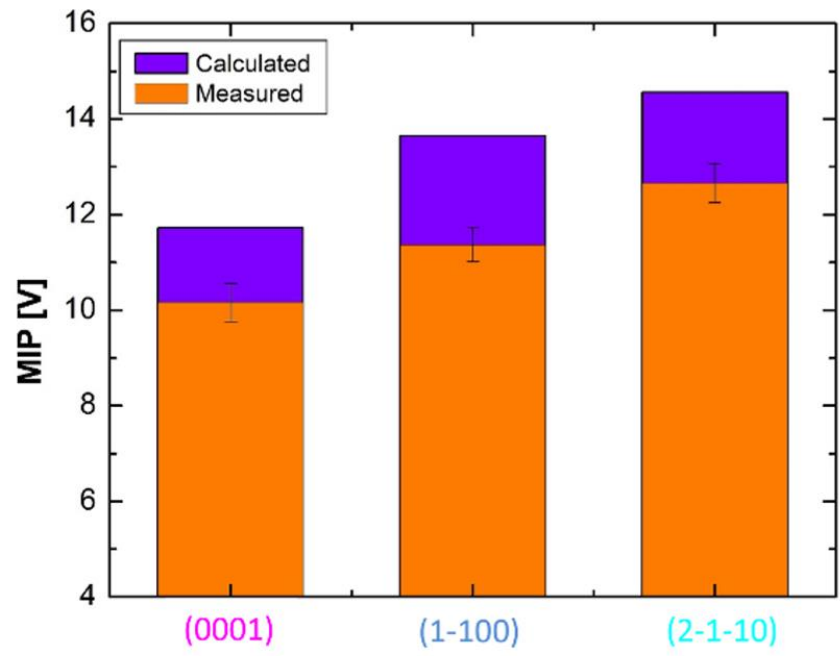

D

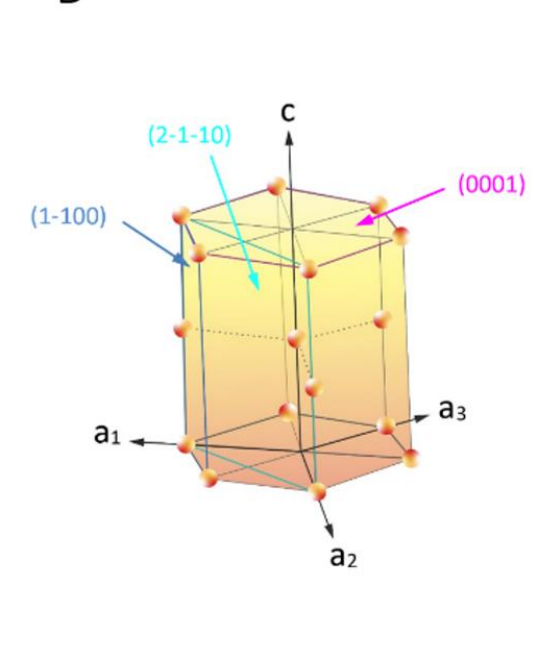

Graphite crystallographic surface orientations

Figure 2. Fig.2 (A) Reconstructed electron phase map from the edge of the first HOPG wedge sample with the (0001) plane at base surface and nominal wedge angle of $30^{\circ}$ (phase spacing: $2 \pi$ ), (B) Unwrapped phase map of (A), (C) Measured and calculated MIP of Bernal graphite for three crystallographic planes, (D) Bernal graphite structure indicating the relevant planes.

\section{References}

[1] M. O'Keeffe, J. C. H. Spence, “On the average Coulomb potential $\left(\phi_{0}\right)$ and constraints on the electron density in crystals", Acta Crystallographica Section A. 50 35-45 (1994).

[2] P. D. Esquinazi, Y.V. Lysogorskiy, Basic Physics of Functionalized Graphite, (Springer International Publishing, 2016), pp. 145-179.

[3] M. Zoraghi, J. Barzola-Quiquia, M. Stiller, P. D. Esquinazi, I. Estrela-Lopis, Influence of interfaces on the transport properties of graphite revealed by nanometer thickness 
reduction, Carbon 139, 1074-1084 (2018).

[4] Avi Auslender, Mahdi Halabi, George Levi, Oswaldo Diéguez, Amit Kohn "Measuring the mean inner potential of $\mathrm{Al}_{2} \mathrm{O}_{3}$ sapphire using off-axis electron holography”, Ultramicroscopy. 198, 18-25 (2019).

[5] P. Stamenov, J. M. D. Coey, Magnetic susceptibility of carbon - experiment and theory,

J. Magn. Magn. Mater. 290-291, 279-285 (2005).

[6] N. B. Brandt, S. M. Chudinov, Ya. G. Ponomarev, Modern Problems in CondensedMatter Sciences, Semimetals - Graphite and its Compounds, (Elsevier, 1988), pp. 175-

196.

[7] J. Heremans, C. H. Olk, D. T. Morelli, Magnetic Susceptibility of Carbon Structures, Phys. Rev. B. 49, 15122-15125 (1994). 